\title{
Using natural-language responses in microcomputer research
}

\author{
CHARLES F. GETTYS, PAUL F. MCKANE, and LYNDON BERGLAN \\ University of Oklahoma, Norman, Oklahoma
}

\begin{abstract}
A microcomputer natural-language interface is described that will identify limited naturallanguage input, such as that obtained in psychological research. In the task studied, subjects made natural-language requests involving 13 DOS commands. A Bayesian algorithm is used to calculate a vector of 13 probabilities expressing the relative likelihood of various DOS tasks that the user was attempting to specify from keywords extracted from the user's input. The algorithm's first-guess accuracy in the limited domain studied is approximately $90 \%$; it will identify the user's request with $99 \%$ accuracy in its first three guesses. This performance is considerably better than that of human experts performing the same task with the same input. Also discussed are issues of cross-validation, robustness, conditional independence, and the calibration of the algorithm's probabilistic output.
\end{abstract}

Computer comprehension of natural language in the most general sense is perhaps the most challenging task that faces the artificial intelligence community (Barr \& Feigenbaum, 1981). However, as this paper will illustrate, it is possible to construct a limited natural-language interface that can identify the user's intentions from naturallanguage responses to problem statements.

Natural-language responses are becoming more commonly employed in cognitive research as the interests of researchers shift to higher level cognitive processes (e.g., Gettys, Pliske, Manning, \& Casey, 1987; Newell \& Simon, 1972). However, manual data reduction of these types of verbal responses has remained labor intensive. Most experimenters, ourselves included, find interpreting, classifying, and counting these responses to be a tedious task, particularly because these verbal responses must be examined by several judges to assess interrater reliability. Furthermore, these analyses are typically performed after the data are collected; it is usually impossible for software to change the conditions of the experiment in real time in response to complicated verbal input.

Our goal, therefore, was to find a technique to enable a microcomputer program to respond to constrained naturallanguage input in real time so that the power and flexibility of verbal input can be exploited. This natural-language interface should be usable by cognitive researchers who are not familiar with the intricacies of natural-language interfaces, and it should be usable on the typical laboratory computers employed by these researchers without inordinate requirements for memory or computational speed.

Correspondence should be addressed to C. F. Gettys, Department of Psychology, University of Oklahoma, Norman, OK 73019 (e-mail: cgettys@oupsy.psy.uoknor.edu).

-Accepted by previous editor, N. John Castellan, Jr.
There are several existing approaches that allow a computer program to respond to constrained natural language in real time. It is important to note that these types of natural-language interfaces are successful because input to the interface is quite constrained by the situation. The subject's natural-language request is made in a constrained environment or using a constrained language. Similarly, in cognitive psychology, subjects respond to stimuli or problem statements, and these stimuli or problem statements constrain the variety of subject responses, making the decoding of natural language much easier.

\section{Types of Constrained Interfaces}

One type of natural-language approach, called the userderived interface, employs small subsets of English needed for computer games, mail systems (Good, Whiteside, Wixon, \& Jones, 1984), and checkbook (Ford, 1981) and calendar management software (Kelley, 1984). This empirical approach exploits the limited domains of these constrained applications and often improves the interface by iteratively extending a parser to handle input that caused parser failures. However, this approach is limited in utility because of frequent syntax errors (Hershman, Kelly, \& Miller, 1979) or other parser errors. For example, Good et al. (1984) were able to iteratively improve their parser so that it accepted over $75 \%$ of their user's commands, but the final performance of their interface was still far from perfect.

A second approach exploits naturally occurring language subsets that are associated with a domain or a profession, such as medicine, meteorology, or the stock market (Harris, 1968; Kittredge, 1982). In these cases, professional communication conventions constrain the variety of user inputs. The user is expected to use a special language, and, as long as the user does so, most of the user's input can be understood. 
There is a general-purpose natural-language interface construction package called LIFER (Hendrix, 1977). This interface development package allows software engineers to create a natural-language interface for their application. The user of this package can construct a natural-language interface for their application in an interval ranging from several days to several months, depending on the complexity of the application (Barr \& Feigenbaum, 1981).

A completely different approach, the so-called "Wizard of $\mathrm{Oz}$ " technique, involves putting a concealed human in the software loop, thus ducking the difficult task of constructing a natural-language interface in software. All natural-language input is sent to the human, who reads the input and responds appropriately to it in real time (Guindon, 1991). The major disadvantages of this approach are that (1) it wastes the experimenter's time-it is usually necessary to invest $1 \mathrm{~h}$ of the experimenter's time for every subject hour-and (2) the response time of the Wizard tends to be slow.

Our review of this literature suggests that it is impractical for many psychological researchers to exploit these software tools or approaches in their laboratories for research purposes. Laboratory computers typically used by research psychologists lack the speed and the memory capacity to run the existing natural-language software. Furthermore, many psychological researchers lack the expertise in natural language required to create their own natural-language software or to adapt existing software to their purposes. What is needed is simple software that will do the limited job needed by the cognitive researcher using a typical laboratory computer. It should not involve an inordinate investment of the researcher's time.

\section{A Constrained Natural-Language Interface}

We needed a constrained natural-language interface that would run on personal computers for a project on adaptive interfaces. An adaptive interface makes an estimate of the user's general expertise and knowledge (Vaubel \& Gettys, 1990) and uses this information to adjust itself to the user. This research on adaptive interfaces suggested that novices would find a natural-language interface useful in enabling them to express their goals in natural language. Once the adaptive interface understands the goal that the user is trying to accomplish, it can help with the details.

Our approach was to create a user-derived interface. The algorithm that we chose searches the subject's naturallanguage input string for keywords and then calculates the Bayesian posterior probabilities for the various goals given the keywords found in the input string. All words found in the input strings are candidates for keywords in this empirical approach.

We used a large number of natural-language strings that varied in the goals they expressed. The frequency with which each word occurs in the natural-language strings was tabulated for each goal, creating a matrix of frequencies for each keyword for each goal. These frequencies were used to estimate conditional probabilities as follows. Assume that an entry in the frequency matrix is $f\left(K_{i}, G_{j}\right)$, where $K_{i}$ stands for the $i$ th keyword, and $G_{j}$ stands for the $j$ th goal. These frequencies can be converted to estimates of conditional probabilities, $p\left(K_{i} \mid G_{j}\right)$, by dividing each $f\left(K_{i}, G_{j}\right)$ by the total number of keywords produced for that goal.

The Bayesian calculation uses these $p\left(K_{i} \mid G_{j}\right)$ conditional probabilities and is quite simple if expressed in vector notation. Suppose we have two vectors: $\mathbf{X}=\left[x_{1}\right.$, $\left.x_{2}, \ldots x_{n}\right]$ and $\mathrm{Y}=\left[y_{1}, y_{2}, \ldots y_{n}\right]$. If we multiply each element in each vector by the corresponding element in the other vector, we produce a third vector, $\mathbf{X}_{-}^{*} \mathbf{Y}=\left[x_{1} y_{1}\right.$, $\left.x_{2} y_{2}, \ldots x_{n} y_{n}\right]$, where $*$ is a pairwise multiplication operator. Using this notation, the iterative form of the oddslikelihood version Bayes' theorem (assuming conditional independence, an important assumption, discussed below), is

$$
\mathbf{V}_{\text {Posterior }}=\mathbf{V}_{\text {Prior }} * \mathbf{V}_{p(K \mid G)},
$$

where $\mathbf{V}_{\text {Posterior }}$ is the desired posterior odds vector, $\mathbf{V}_{\text {Prior }}$ is the prior odds vector, and $\mathbf{V}_{p(K \mid G)}$ is a likelihood vector of $p\left(K_{i} \mid G_{j}\right)$ probabilities for the $i$ th keyword being processed.

This algorithm is used to iteratively calculate posterior odds for the first keyword in the string by pairwise multiplying the prior odds vector by the vector of conditional probabilities from the matrix for that keyword. For the first calculation, the prior odds vector is set to numbers that are proportional to the prior probabilities of the goals. Succeeding calculations for additional keywords are calculated by substituting the posterior odds vector for the prior odds vector and repeating the calculation for the next keyword. The initial prior odds vector can be estimated by the experimenter from relative frequencies, or uniform prior odds can be used (all odds are set to one) with little loss in efficiency (see Edwards, Lindman, \& Savage, 1963).

The following simplified Pascal code fragment illustrates the heart of the algorithm:

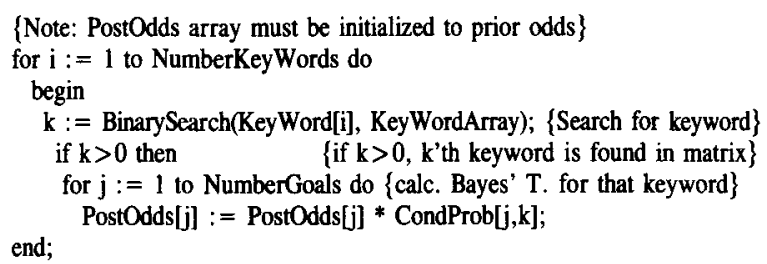

Several comments should be made. First, some may find the term "odds" confusing, since it is usually applied to two alternatives rather than many; however, our use of "odds" to refer to many alternatives is a straightforward generalization of the pairwise odds concept. Second, when estimating the matrix of conditional probabilities, $p\left(K_{i} \mid G_{j}\right)$, a number between 0.01 and 1 should be substituted for any zero frequencies, because using a zero $p\left(K_{i} \mid G_{j}\right)$ probability in a Bayes calculation means that the corresponding posterior odds will be zero. This obviously is undesirable. Finally, for many uses it is desirable to normalize the posterior odds vector to a vector of posterior probabilities by dividing each odds term by the sum of that vector.

The purpose of the present study was to investigate the accuracy of the Bayesian algorithm and variables that affect its accuracy. We collected natural-language strings 
from a large group of subjects to estimate the $p\left(K_{i} \mid G_{j}\right)$ matrix. Once this matrix was created, the strings were used to assess the accuracy of the algorithm.

\section{METHOD}

\section{Subjects}

The subjects were 77 college students recruited from the introductory psychology subject pool with the stipulation that they should be able to type at least 10 words per minute. Most subjects evaluated their computer expertise as being in the low to moderate range.

\section{Instructions}

Each subject was asked to play the role of a novice computer user who was giving directions to an experienced computer user, Susie, who was operating her computer on the subject's behalf. The instructions emphasized that these requests should be made in the subject's natural language: "Your job is to tell Susie, in your own natural English, the tasks that you want to accomplish on her computer."

\section{Problem Statements}

Our adaptive interface helps users use DOS by helping the subjects use 13 simple DOS commands, which were cd, copy, date, del, dir, fc, format, label, md, rd, ren, tree, and type. We wrote two problem statements for each DOS command, giving a total of 26 problem statements. The technique of writing the problem statements was developed in several pilot studies. We found that indirect wording of the problem statement, such as "How would you tell Susie to do this?" was crucial to prevent the subjects from simply restating prose from the problem statement. A file, probset.txt, in the distribution disk contains the complete text of all problem statements. The following are examples of the two problem statements for the copy command:

If you have spent a lot of time working on a term paper, you often make a duplicate copy of that paper to prevent its loss by accident. With computers, this situation occurs frequently. You need to copy your file from Susie's computer to your disk in case Susie accidentally erases the copy on her computer. How would you tell Susie to do this?

It is usually a good idea to make a second, or "backup" copy of your most important files usually on a completely different diskette. How would you tell Susie to do this for you?

Examples of the first 10 natural-language requests that the subjects made to the first of the two above problem statements are as follows:

"copy my file so I won't lose it"

"COPY DISK"

"Please copy this file from the hard drive onto my diskette."

"I need to copy my file from your computer to my disk."

"Please copy this file."

"Susie copy my files"

"copy my paper onto my disk"

"Susie save the copy on the sceen [sic] onto the diskette."

"Susie, I need to make a copy of my files to prevent them from being erased."

"please record all data on disk"

It is interesting to observe that although the keywords copy and file occur frequently, no particular keyword occurs in every string. Furthermore, even though the problem statement constrains the language greatly, there still is considerable diversity in the way the subjects could choose to phrase their requests. Finally, some commands are technically in error, such as "COPY DISK."

\section{Data Collection Program}

Natural-language strings were collected from the subjects using a Turbo Pascal program, Natural6. After reading the instructions, the subjects read a series of problem statements that explained in general terms the computer goal that they should try to achieve. For each problem statement, the subjects made a natural-language request expressing that goal to a hypothetical computer user, Susie, by typing that request on the keyboard. Using the keywords found in the input string, an algorithm calculated the goal; if the goal was among the three most probable goals according to the algorithm, the subject was allowed to move on to the next problem statement. Otherwise, the subjects were encouraged to rephrase their requests and try again. Up to three such requests were made for each problem statement before moving to the next.

\section{Procedure}

The subjects entered their requests at their own pace for the 26 problem statements presented in a randomized order. All subjects finished the experiment in less than $50 \mathrm{~min}$, and the fastest subject finished in $18 \mathrm{~min}$. The data of interest that resulted from this procedure were 2,002 strings ( 77 subjects $\times 26$ problem statements). We examined only the subject's initial response to each problem statement. Second and third attempts were included in the procedure primarily for motivational reasons to encourage the subjects to form their requests carefully.

\section{RESULTS AND DISCUSSION}

\section{Quick and Dirty Approaches}

Prior to the study described above, we performed several pilot studies to learn more about the problem that we confronted. These were informal studies, using different subjects and procedures, and their details are not important. However, we did learn several interesting things: Our first pilot study explored the feasibility of choosing keywords and estimating the relationships between keywords and goals on intuitive grounds. We failed miserably. Our intuitions were rarely correct regarding which keywords should be chosen or what the relationships between keywords and goals should be. For example, it matters whether a keyword is singular or plural, conjunctions are important, and many important keywords are not computer terms.

We decided to use an empirical approach for our second pilot study, since the intuitive approach, in our hands at least, seemed to have little promise. This study was primarily devoted to refining the problem statements for the reasons discussed above. Its results showed promise. The frequencies obtained in the second pilot from 10 subjects were used in the algorithm employed in the main study.

\section{Overall Performance}

All 2,002 strings obtained from the subjects in the main study were used to construct an $f\left(K_{i}, G_{j}\right)$ matrix. All distinct words were keywords in this analysis. Since there were 13 possible goals in our study that were equally likely, we used a uniform prior odds vector, where all terms were set to one.

To assess the accuracy of the algorithm, we then extracted the keywords in each string and used the $f\left(K_{i}, G_{j}\right)$ matrix in a Bayesian calculation to produce a posterior probability vector. Our data analysis software examined that vector to see if the DOS command (or, more generally, the subject's goal) could be accurately inferred from the probability vector.

The accuracy of the algorithm can be assessed by examining the vector of posterior probabilities and guessing 
Table 1

Factors Influencing the Accuracy of the Algorithm

\begin{tabular}{|c|c|c|c|}
\hline \multirow[b]{2}{*}{ Conditions } & \multicolumn{3}{|c|}{ Number of Guesses } \\
\hline & First & Second & Third \\
\hline A. Baseline performance (708 keywords) & .921 & .980 & .993 \\
\hline B. Keywords reduced to 264 & .893 & .965 & .984 \\
\hline C. Cross-validated by dropping subject's data & .850 & .949 & .979 \\
\hline D. Assuming conditional independence & .898 & .967 & .986 \\
\hline E. Exploiting conditional dependence & .904 & .974 & .990 \\
\hline F. Keyword pairs with order preserved & .912 & .974 & .996 \\
\hline G. Matrix spelling corrected only & .898 & .967 & .986 \\
\hline H. Performance of self-proclaimed experts & .872 & .966 & .979 \\
\hline I. Performance of remainder of subjects & .895 & .966 & .989 \\
\hline J. Problem data removed from matrix & .922 & .972 & .987 \\
\hline K. Subject and problem data removed & .815 & .935 & .970 \\
\hline L. 0.5 used for zero cells rather than 0.01 & .883 & .958 & .976 \\
\hline M. Performance of experimenter CFG & .744 & .782 & .833 \\
\hline N. Performance of experimenter PFM & .730 & .885 & .910 \\
\hline O. Performance of experimenter LB & .833 & .923 & .961 \\
\hline P. Performance of DÓS expert & .623 & .782 & .833 \\
\hline
\end{tabular}

the goal or problem statement that the subject was expressing. This analysis exploits the ordinal information contained in the posterior probabilities. A first guess can be made by inferring that the problem statement that the subject was responding to is the problem statement receiving the highest posterior probability. The accuracy of the first guess, therefore, is the relative frequency with which the first guess is correct. It is possible to calculate the accuracy of two or three guesses using similar logic.

Table 1 shows the cumulative relative frequency for the first three guesses and expresses the accuracy of the algorithm when you allow it a first guess, two guesses, or up to three guesses if necessary. As can be seen from examining row A of Table 1, the first guess was correct $92.1 \%$ of the time, the first two guesses were correct $98 \%$ of the time, and first three guesses include the correct DOS command $99.1 \%$ of the time. This level of accuracy is better than we needed for our adaptive interface, and it should be sufficient for most uses.

\section{Reducing the Number of Keywords}

The Turbo Pascal code that implements our algorithm is designed to use memory efficiently. The matrix used by the algorithm is stored as records on the heap. It uses 64 bytes for each keyword record. However, the size of this matrix may produce a problem for some users whose experimental software already uses most of the available memory. For example, the baseline performance shown in row A of Table 1 is based on 708 keywords, and the keyword matrix occupies 45,312 bytes on the heap. For our adaptive interface, memory usage should be minimized. For this reason, we developed a method of screening the keywords for relevance. We computed the chisquared statistic on the $\mathbf{f}\left(\mathbf{K}_{\mathbf{i}}, \mathbf{G}_{\mathbf{j}}\right)$ vector for each keyword. If the chi-squared value is greater than an arbitrarily chosen critical value, such as 20 , the keyword is retained in the matrix; otherwise, it is dropped. Row A of Table 1 was produced with a criterion value of zero; all words used by the subjects were keywords, even though such words as Susie were used with approximately equal frequency for all problem statements. Row B of Table 1 shows the performance of the algorithm with the chisquared criterion of 20 , which uses 264 keywords and 16,896 bytes on the heap. As can be seen by comparing rows $A$ and $B$, a two-thirds reduction in the number of keywords (and memory used) degrades the accuracy of the algorithm only slightly. In all analyses reported below row A of Table 1, we used a chi-squared criterion of 20.

\section{Cross Validation}

Rows A and B overestimate the performance of the algorithm somewhat because the matrix contains data from the subject whose performance is being assessed. Therefore, that person's idiosyncratic vocabulary and way of phrasing natural-language requests may be exploited by the algorithm. In normal use, the matrix would be obtained from one group of subjects and would be expected to work for a second independent group. We can estimate the performance of the algorithm in actual use by temporarily dropping the data of each subject whose data are being analyzed in turn from the matrix. The results of such a calculation are shown in row $\mathrm{C}$ of Table 1 . As can be seen by comparing rows $B$ and $C$ in Table 1 , the firstguess accuracy drops about $4 \%$ when cross-validated, still yielding acceptable accuracy.

\section{Robustness and the Conditional Independence Assumption}

Using Bayes' theorem for practical purposes usually involves assuming conditional independence. In this case, it is convenient to assume conditional independence among keywords. For example, $p\left(K_{i} \mid G_{j}\right)$ should equal $p\left(K_{i} \mid G_{j}, K_{l}\right)$ for $i, j$, and $l$ (see Steiger \& Gettys, 1973, for more detail). This assumption probably is only rarely met in practice, and the important question to be addressed is the impact of any conditional dependencies that may be present.

We assessed the impact of possible conditional dependencies by performing two parallel calculations. One calculation was performed using the techniques that assume conditional independence (as previously described). A second calculation that can exploit pairwise conditional dependencies was also performed. This analysis used single keywords and pairs of keywords. Since the definition of what constitutes a keyword is arbitrary, a pair of keywords can serve as a keyword in the algorithm. For example, the matrix contained the single keywords delete and file and the compound keyword delete-file. If all possible pairs of keywords in an input string are used, a combinatorial explosion of the number of keywords results; we therefore screened all keywords (single and compound) for relevance by using a chi-squared value of 20 . Our second algorithm used the compound keywords, if they existed. If the compound keyword was found, it was used in the Bayesian calculation, and the corresponding single keywords were dropped. If the compound keyword was not found, then the single keywords were used. Rows D and 
E of Table 1 show the results of these two analyses. The single-keyword analysis assuming conditional independence is shown in row D. (Row D differs from row B in that keyword spelling has been corrected in row D.) The keyword-pair analysis that can exploit conditional dependence is shown in row $\mathrm{E}$. As can be seen from inspection of rows $\mathrm{D}$ and $\mathrm{E}$, a calculation that exploits conditional dependencies performs only slightly better. Furthermore, the number of keywords increased from 264 to 1,348 , and heap memory usage increased from 16,896 to 86,272 bytes. The time to calculate the algorithm increased from .069 to $.152 \mathrm{sec}$ per string, using a $20-\mathrm{MHz}$ 386 computer.

However, if the algorithm uses keyword pairs, it can exploit word-order information, which is important in English. For example, "The dog bit the boy" and "The boy bit the dog" have quite different meanings. The software that creates the matrix sorts the keywords into alphabetical order before forming pairs of keywords, thus destroying order information but minimizing the number of different keyword pairs. Order can be preserved by setting a software switch that prevents a call to the sort routine. Many more potential keyword pairs will result, but some will not survive chi-squared screening procedure in matrix construction. A keyword-pair analysis that preserved order used 1,678 keywords, and the matrix occupied 107,392 bytes on the heap. The accuracy of the first, second, and third guesses is shown on row F of Table 1 . These results can be compared to row $\mathrm{E}$ of Table 1. Preserving order resulted in a slight gain in accuracy, but the gain is not enough to justify the increased memory usage of ordered keyword pairs in our situation. Other users, where memory usage is not important, or where order information is crucial, may choose to use this version of the algorithm.

\section{Spelling Errors}

We counted 134 spelling errors; the most frequent error was a misspelling of "separate." Spelling errors may reduce the accuracy of the Bayesian algorithm because, if a word is misspelled, it may not be identified as a keyword. If spelling errors are a problem with the population of subjects used, a memory-resident spelling checker, such as Borland's "Lightning," can be used. We explored correcting the spelling during matrix construction and found that it made little difference in the performance of the algorithm. Row $\mathbf{G}$ in Table 1 resulted from correcting keyword spelling during matrix construction, but not from correcting the input strings. It resulted in only a slight improvement over row B, where no spelling corrections were made.

\section{Subject Expertise}

When examining the subjects' natural-language requests, we noticed that a few subjects used what might be termed "pseudo-DOS," and there were clear individual differences in the expertise with which requests were phrased. As part of the instructions, we asked subjects to rate their expertise with DOS using a 7-point Likert scale. We classified those subjects who rated themselves between 5 and 7 as self-proclaimed experts. The performance of the algorithm for these 10 subjects is shown in row $\mathbf{H}$ of Table 1 , and the performance of the remaining 67 subjects is shown on row I. As can be seen from examination of rows $\mathrm{H}$ and $\mathrm{I}$, the algorithm works quite well for both groups of subjects and apparently is not sensitive to subject expertise. We recognize, of course, that selfreports of expertise are not definitive, and the range of expertise was probably restricted in our sample. Nevertheless, it is interesting that both groups performed about the same.

\section{Exploration of Boundary Conditions}

The reader may wonder, as we did, about the extent to which the success of the algorithm is tied to the particular problem statements. It is clear that problem statements shape the way subjects phrase their requests. How well does the algorithm work if the subject is responding to a problem statement not used in constructing the matrix? Using logic similar to that employed in the cross-validation calculations discussed earlier, we dropped the frequency counts attributable to each problem statement from the matrix when we analyzed the requests made in response to that problem statement. The matrix was then restored to its original values, and data from the next problem statement was analyzed using the same procedure. For example, the algorithm was calculated for all requests to the first COPY problem statement without using data from that statement. The algorithm can still perform because data from the second COPY problem statement is still present in the matrix. The results of this analysis are shown in row $J$ of Table 1 . There was no apparent degradation of performance resulting from temporarily dropping the problem statement information from the matrix. In fact, this analysis shows slightly better performance, which puzzles us.

It is possible, of course, to drop both the data of a particular subject and the problem data from the matrix simultaneously; this result is shown on row $\mathrm{K}$ of Table 1 . Row $\mathrm{K}$ is an estimate of how well one might expect the algorithm to work in a research situation in which new subjects are used and they are responding to problem statements that were not used in constructing the matrix. As can be seen, there is a noticeable drop in accuracy. This defines a boundary condition for our study: We do not know how well the algorithm will work in the absence of any problem statements.

\section{Calibration of Probabilities}

The analyses that have been discussed so far exploited the ordinal properties of the Bayesian posterior probabilities. However, the success of some possible applications for the algorithm depends on the magnitude of the probabilities. The extent to which environmental frequencies match the posterior probabilities is called calibration in the decision-making literature (Yates, 1990, p. 45), and the logic of a calibration analysis is simple. Posterior probability estimates of event frequencies are compared with 


\section{Calibration}

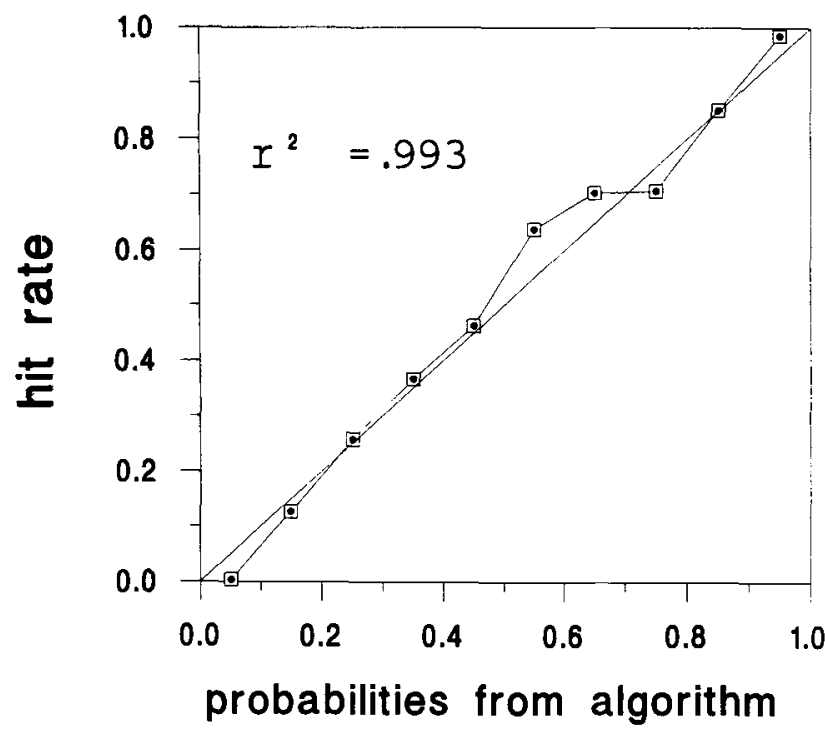

Figure 1. Calibration graph for Bayesian probabilities produced by the natural-language algorithm. Perfect calibration is found when all data points fall on the diagonal.

the relative frequencies with which events occur. The probabilities are said to be well calibrated if there is a good match.

The calibration of the algorithm is slightly affected by the value substituted for zero frequencies in the $f\left(K_{i}, G_{j}\right)$ matrix. We explored the calibration of the algorithm using various substitution values for zero frequencies ranging from 0.001 to 2.00 . (A value of 0.01 was used in all previously reported analyses.) The best tradeoff between ordinal accuracy and calibration was found to be a substitution value of 0.5 . Using this value, we obtained good calibration, as well as good ordinal accuracy (as can be seen by comparing Rows D and L of Table 1). The calibration data are shown in Figure 1. If the algorithm were perfectly calibrated, all points would be found on the diagonal line. As can be seen by inspecting Figure 1, the probabilities from the algorithm are well calibrated; the $r^{2}$ for these data is .993 .

\section{Algorithms for Displaying Guesses for Confirmation}

It is fortunate that the algorithm is well calibrated because this allows the construction of an additional intelligent algorithm for showing the subjects the results of the Bayesian algorithm for confirmation. It is a truism that software users dislike software that is a juggernaut. For this reason, the output of the natural-language algorithm should be fed to a second algorithm that displays the Bayesian algorithm's best guesses as to what they are actually requesting. The subject can confirm one of these guesses or request that additional guesses be shown for possible confirmation. One could simply show the three best guesses, but we suggest the following algorithm be employed: First, rank order the goals according to the posterior probabilities. Second, display the goals in the order of most probable to least probable until the cumulative sum of the probabilities for the displayed alternatives is equal to or greater than some value, such as $\mathbf{0 . 9 5}$.

We have used this algorithm in demonstration software, and it works well. Most of the time, only one alternative is displayed for confirmation. Sometimes two or more alternatives are shown, and it is easy for the subject to scan this list since it almost always has three or fewer alternatives on it. If the subjects feel that the list does not contain a goal that matches their requests, they may display all of the alternatives.

\section{Wizard of $\mathrm{Oz}$ Versus the Bayesian Algorithm}

As discussed previously, an alternative to the Bayesian algorithm is the Wizard of $\mathrm{Oz}$ technique. We simulated this technique to estimate the accuracy of human "Wizards" in our situation. We used ourselves as subjects on the grounds that we were the most knowledgeable possible Wizards, being intimately familiar with the problem statements and quite familiar with the requests that our subjects made. Our performance was expected to exceed that of less knowledgeable Wizards, particularly Wizards who had not been previously immersed in the subjects' problem statements. Thus, we believed that using ourselves as Wizards would give upper bound estimates of typical Wizard accuracy, where typical Wizards who had not had our experience would have somewhat lower accuracy.

Each of the experimenters were shown 78 strings randomly chosen ( 3 from each of the 26 problem statements) from the 2,002 strings we collected from the subjects. Each of us made a first, second, and third guess for each string, and we each spent about $1 \mathrm{~h}$ making our guesses. The results are shown in rows $\mathrm{M}-\mathrm{O}$ in Table 1 . As can be seen by inspecting these results, the algorithm is noticeably better than all 3 human Wizards and considerably better than two of the Wizards. Row P contains data produced by a DOS "super user" of our acquaintance who performed the same task as we did. This highly competent person used DOS on a daily basis. His data suggest that we were in fact very knowledgeable Wizards, and his data probably are more typical of a Wizard who is unacquainted with typical user responses.

These results were quite surprising. Our initial expectation was that human experts would be better than the algorithm; we wanted to know how much better. We examined our errors and discovered that about one third of our misses were based on erroneous input from the subjects. In other words, the subjects were occasionally making bad requests that any reasonable expert would misinterpret, such as "erase files" where "copy files" would be correct for that problem statement. (In these cases, both the algorithm and the expert would make an error.) Our data as experts were based on subsets of 78 of the 2,002 strings, 
and we had no desire to examine all 2,002 strings to estimate the number of bad requests. Instead, we output all strings for which the algorithm's first guess was in error, and each of us examined these strings independently. Of the strings we examined, we concluded that 107 were completely in error, or about $5 \%$ of the 2,002 strings. If these strings are excluded from the analysis, the firstguess hit rate of the algorithm is estimated to be about 0.94 . The expert accuracy figures could be corrected using the same logic. Of course, it is possible that the algorithm succeeds occasionally with a completely erroneous string because of a fortuitous combination of keywords; therefore, $94 \%$ is only a rough estimate of the accuracy of the algorithm when the subject's request makes sense.

\section{GENERAL CONCLUSIONS}

We have created a natural-language interface that will work in our application with acceptable accuracy, speed, and memory usage. This interface should be easy to use by nonexperts; it responds rapidly and does not use inordinate amounts of memory.

The reader may wonder if the techniques described here could be "scaled up" to a much larger situation. Our intuition suggests that this would be infeasible. One could increase the number of "goals" from 13 to about 20 without boring the subjects too badly, and we would guess that acceptable accuracy might be found with 20 goals. Beyond this, we emphatically recommend use of the software supplied with the package to calculate the accuracy of the algorithm to see if it is acceptable.

Potential users should evaluate our approach to see if it suits their application and should always gather enough data to assess the accuracy of the algorithm in their situation. It is difficult for us to assess the generality of our approach in the absence of a wide variety of different applications. Our intuition suggests that it will work satisfactorily in most simple situations. The authors plan to keep track of the success of various applications, and potential users should contact us for further information.

As previously discussed, our algorithm in its default form does not exploit word order and therefore would evaluate "The dog bit the boy" identically to "The boy bit the dog." By setting a software switch in the matrix construction software and using pairs of keywords, order information can be exploited, but the user should study his or her application carefully to decide which version of the algorithm, if any, is more suitable.

Finally, the reader should consider the desirability of using keyboard input in his or her experimental situation. Obviously, keyboard input is slow and attention demanding for some subjects. We have dealt with this problem by the routine use of a typing test in our research. Our subject recruitment states that subjects must be able to type 10 (or 20) words per minute, and subjects must pass a typing test to continue in the experiment.
What applications might profit from our approach? Several examples besides our own come to mind. Some complicated experimental programs need a help system, and help systems are natural candidates for a natural-language interface such as ours. Research on problem solving sometimes involves software simulations of machines. Often the subjects have to be taught command vocabularies for the machine before data collection can start. A naturallanguage interface that could identify the subject's intention might be useful. Computer research with children who can not be expected to learn a command syntax could also be aided.

\section{Software Availability}

The software was written in Borland's Turbo Pascal 6.0 in such a way as to make "off-the-shelf" application relatively easy for the potential user. Users declare Turbo Pascal units "NatConst" and "Natural" at the beginning of their program. Calls to four procedures in "Natural" then implement the natural-language interface. One procedure loads the matrix on the heap. A second procedure accepts string input from the program, identifies keywords in that string using a binary search of the keyword array, and calculates the algorithm for each keyword. A third procedure accepts the output from the algorithm and displays choices to the subject for confirmation. The fourth procedure loads the confirmation choices into memory.

In addition to this software, there is a series of utility programs for the collection of natural-language input needed to build the matrix, to construct the matrix itself, and to evaluate the accuracy of the algorithm. All programs read ASCII files to particularize the application for the user. We estimate that a decent Pascal programmer who has many personal computers available for data collection could have a working version of the interface in less than $40 \mathrm{~h}$ of effort, where the biggest expenditure of time would be data collection. The software package is approximately $444 \mathrm{~K}$ in size and contains 29 files. We recommend that interested users get a distribution disk from us, rather than asking us to e-mail the software. The Pascal source code for this package can be obtained by sending a check for $\$ 10$, payable to Decision Processes Laboratory/Oklahoma University, to the senior author to defray the costs of reproduction and mailing. Further information may be obtained by contacting the senior author.

\section{REFERENCES}

Barr, A., \& FeIGenbaum, E. A. (EDs.). (1981). The handbook of artificial intelligence (Vol. 1). Los Altos: Kaufmann.

Edwards, W., Lindman, H., \& SAvage, L. J. (1963). Bayesian statistical inference for psychological research. Psychological Review, 70, 193-242.

FORD, W. (1981). Natural language processing by computer $-A$ new approach. Unpublished doctoral dissertation, Johns Hopkins University, Baltimore, Maryland.

Gettys, C. F., Pliske, R. M., Manning, C., \& Casey, J. T. (1987). An evaluation of human act generation performance. Journal of $\mathrm{Or}$ ganizational Behavior \& Human Decision Processes, 39, 23-51. 
Good, M. D., Whiteside, J. A., Wixon, D. R., \& JoNes, S. J. (1984). Building a user-derived interface. Communications of the ACM, 27 1032-1043.

GuinDon, R. (1991). Users request help for advisory systems with simple and restricted language: Effects of real-time constraints and limited shared context. Human-Computer Interaction, 6, 47-75.

HARRIS, Z. S. (1968). Mathematical structures in language. New York: Wiley.

HENDRIX, G. G. (1977). Human engineering for applied natural-language processing. International Joint Conferences on AI, 5, 183-191.

Hershman, R. L., Kelly, R. T., \&iller, H. G. (1979). User performance with a natural-language query system for command control (Rep. No. NPRDC-TR-79-7). San Diego, CA: Navy Personnel Research and Development Center.

KeLLey, J. F. (1984). An iterative design methodology for user-friendly natural-language office information applications. ACM Transactions on Office Information Systems, 2, 26-41.
KITTREDGE, R. (1982). Variation and homogeneity of sublanguages. In R. Kittredge \& J. Lehrberger (Eds.), Sublanguage: Studies of language in restricted domains (pp. 107-137). New York: de Gruyter.

Newell, A., \& Simon, H. A. (1972). Human problem solving. Englewood Cliffs, NJ: Prentice-Hall.

Steiger, J. H., \& Getrys, C. F. (1973). Conditional dependence and decomposition strategies in diagnostic inference systems. Organizational Behavior \& Human Performance, 10, 88-107.

VAUBEL, K. P., \& GeTTYS, C. F. (1990). Inferring user expertise for adaptive interfaces. Human-Computer Interaction, 5, 193-242.

YATES, J. F. (1990). Judgment and decision making. Englewood Cliffs, NJ: Prentice-Hall.

(Manuscript received October 1, 1991; revision accepted for publication November 1, 1993.) 